\title{
TRANSIENT PONDEROMOTIVE EFFECTS IN SUPERCONDUCTING CAVITIES *
}

\author{
T. Powers ${ }^{\sharp}$ K. Davis, TJNAF, Newport News, VA, 23606, U.S.A.
}

\begin{abstract}
A number of experiments were performed on an installed and operational 5-cell CEBAF cavity to determine the minimum time required to re-establish stable gradient after a cavity window arc trip. Once it was determined that gradient could be re-established within 10 ms by applying constant power RF signal in a voltage controlled Oscillator-phase locked loop based system (VCO-PLL), a second experiment was performed to determine if stable gradient could be re-established using a fixed frequency RF system with a simple gradient based closed loop control system. During this test, fluctuations were observed in the cavity forward power signal, the source of which was later determined to be ponderomotive in nature. These ponderomotive induced vibrations were quantified using a cavity resonance monitor and a VCO-PLL RF system. Experimental results, analysis of the resultant klystron power transients, the decay time of the transients, and the implications with respect to fast reset algorithms will be presented.
\end{abstract}

\section{BACKGROUND}

One significant cause of cavity trips in the CEBAF machine is vacuum discharges, or arcs, which occur in the in the region of the cold window. While an individual cavity that arcs every few days does not substantially degrade machine availability, recurring arcs in a reasonable fraction of the $338 \mathrm{SRF}$ cavities installed in CEBAF can negatively impact operations. The goal of this work was to determine the minimum time required before stable gradient could be re-established in a cavity after an arc.

The arc events can be sub-divided into: 1) arcs in the vacuum space between the warm window (attached to the external waveguide) and the cold window (attached directly to the SRF cavity), and 2) arcs on the cavity side of the cold window. The former has a cavity gradient decay time of 1 to $2 \mathrm{~ms}$. The latter has a decay time between $50 \mathrm{~ns}$ and a few hundred microseconds $[1,2]$.

\section{EXPERIMENTAL SETUP}

The intent was to induce arc events using the existing low level RF (LLRF) system, and then reapply RF following a variable delay. The system response would be measured to varying time delays before RF was reapplied under the following conditions. Apply a constant level and frequency drive signal to the cavity. Second, apply a constant power variable frequency signal generated by using the source as a voltage controlled oscillator, in a phase locked loop configuration. The third approach was to apply a fixed frequency variable level RF signal by using the source as a amplitude modulated system with a closed loop gradient control where the sampled signal is the cavity field probe signal after rectification using a crystal detector.

\section{Generation of the Secondary Pulse}

The experiments made use of an RF switching network and combiner to apply a secondary RF source to the existing klystron drive signal, see Figure 1. During normal operation the LLRF module drove the klystron via the summing junction while RF Switch 1 was OFF and RF switch 2 was ON. When an arc fault was detected the klystron drive signal from the LLRF module was turned $\mathrm{OFF}$ and the fault signal triggered the delayed pulse generator. After the desired delay, a pulse was produced

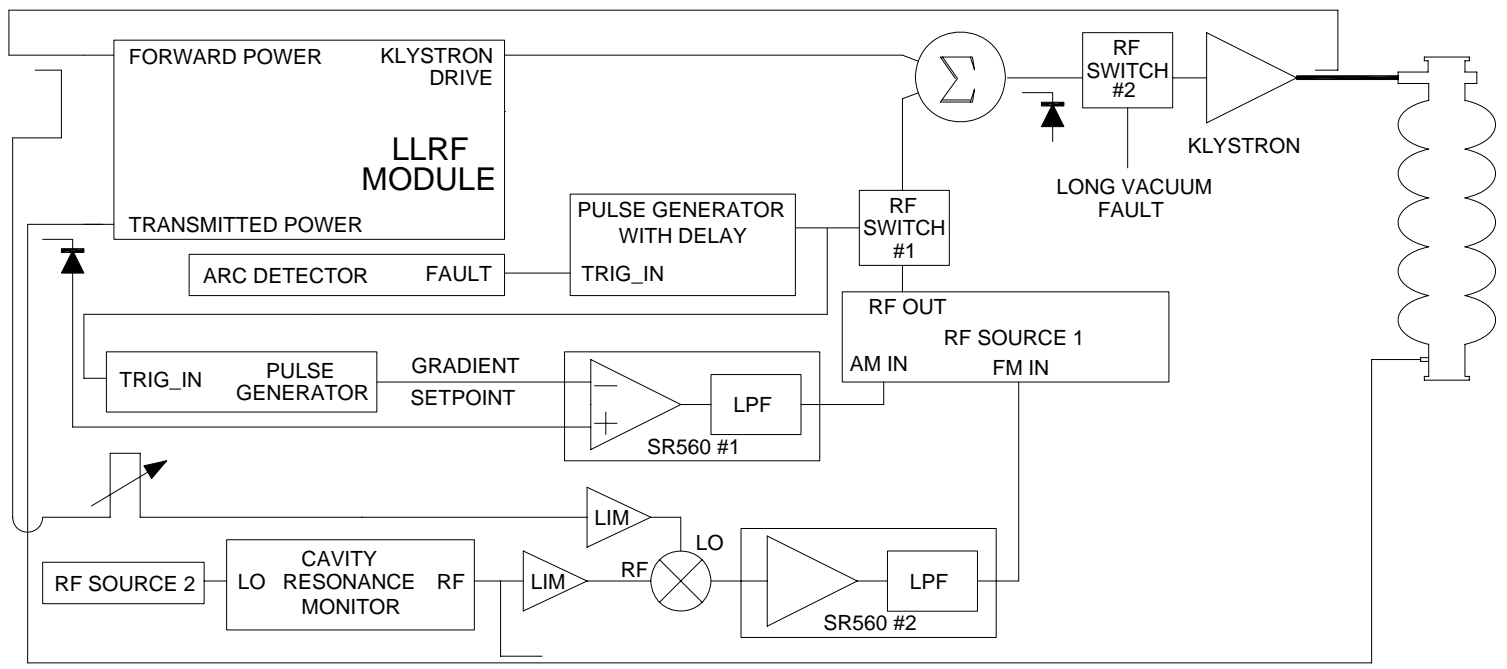

Figure 1: Experimental Setup.

*Supported by US DOE Contract No. DE-AC05-84ER40150

"powers@jlab.org 
that would turn RF switch 1 ON. RF switch 2 was used as a secondary interlock. If a waveguide vacuum fault was detected for longer than $1 \mathrm{~s}$ the drive signal to the klystron would be interrupted. During open loop operation the AM and FM modulation modes were disabled on RF source 1. Phased locked loop control and gradient control were enabled by turning on the FM or AM modulation respectively. A test pulse was generated by applying an external light source to the arc detector which generated a fault signal without producing an actual arc.

The phase loop used a sample of the forward power and the cavity field probe signals to generate a phase error signal. The phase error signal, which was the output of the mixer, was amplified and applied to the FM control input of RF source 1 . The cavity resonance monitor was also used when the system was operated in this mode. The output of the cavity resonance monitor is a DC signal that is proportional to the difference between the cavity frequency and that of RF source 2.

The gradient control loop compared a sample of the transmitted power signal that was rectified using a crystal detector with the output of a secondary pulse generator. The amplitude of the pulse generator was set such that the error signal out of amplifier 1 was equal to zero when the gradient was at the desired value. The output of the amplifier was applied to the AM input of the RF source 1.

\section{EXPERIMENTAL RESULTS}

Two types of arcing events were observed, each type had a characteristic decay time. Additionally, a test pulse was injected to the arc detector system which resulted in an event that had the natural decay time of the cavity. These decay waveforms are shown in Figure 2. In the first type, (A), the arc occurred on the cavity side of the cold window, and the stored energy decayed away in approximately $50 \mu \mathrm{s}$. In the second type, (B) the arc occurred on the waveguide side of the window and the stored energy decayed away in approximately $2.5 \mathrm{~ms}$. The third type of event was the results of triggering the system using a test pulse in the arc detector circuit. In this type of event the decay time was on the order of $5 \mathrm{~ms}$.

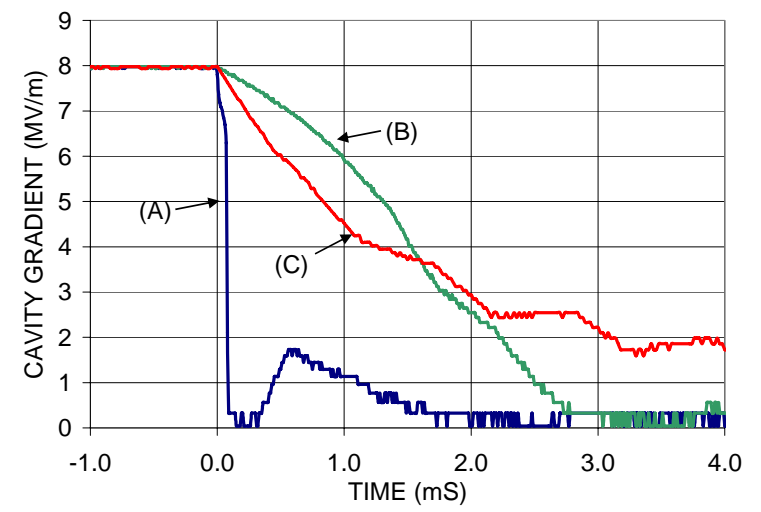

Figure 2: Typical Decay Times for three types of events (A) an arc on the cavity side of the window, (B) an arc on the waveguide side of the cold window and $(C)$ the natural decay of the cavity after a test pulse.
The perturbations in the natural decay measurement were present because the klystron was pulsing at a low level for approximately $50 \mathrm{~ms}$ after the fault.

During previous studies $200 \mathrm{~ns}$ burst of intense gamma radiation were observed when ever arcs on the cavity side of the cold window were recorded [2]. The theory is that gas released from the surface is ionized by the cavity electric field; the free electrons are then accelerated by the cavity, reducing the stored energy. It was uncovered during this work that the sudden decrease in Lorentz force during this rapid gradient loss causes a step input that excites the mechanical vibration modes of the cavity.

\section{Initial Results}

The initial experimental results are fully described in [3]. In that work it was determined that the first series of tests RF source 1 was configured to provide a fixed power at fixed frequency signal. The initial goal was to determine the decay time for the gas that was released into the window vacuum space by the discharge. Once it was determined that the vacuum recovered within $10 \mathrm{~ms}$, a series of closed loop experiments were initiated.

In the second set of experiments attempts were made to restore the cavity gradient after a fault using a closed loop gradient system, i.e. AM modulation was turned on. During that work it was determined that, although the gradient could quickly be re-established for an arc following an event on the waveguide side of the window, excessive transients in the forward power often exceeded the capabilities of the klystron and lead to secondary arcs when the event was on the cavity side of the window.

A VCO-PLL system was used in conjunction with a cavity resonance monitor in order to measure the ponderomotive effects due to the sudden reduction in Lorentz force detuning. The results of that work are summarized in Table 1.

\section{Decay of Induced Vibrations}

The final set of experiments was to determine the decay time of the ponderomotive induced vibrations. With this data one could determine the minimum time required before a cavity could be reset following each type of trip. The system was set up with a closed gradient loop for the

Table 1: Summary of Experimental Results.

\begin{tabular}{|c|c|c|c|}
\hline Event Type & $\begin{array}{c}\text { Gradient } \\
\text { Decay } \\
\text { Time }\end{array}$ & $\begin{array}{c}\text { Gradient } \\
\text { Fill Time }\end{array}$ & $\begin{array}{c}\text { Pk-Pk } \\
\text { Frequency } \\
\text { Shift }\end{array}$ \\
\hline $\begin{array}{c}\text { Background } \\
\text { Microphonics }\end{array}$ & NA & NA & $30 \mathrm{~Hz}$ \\
\hline $\begin{array}{c}\text { VCO-VCO test } \\
\text { pulse }\end{array}$ & $3 \mathrm{~ms}$ & $3 \mathrm{~ms}$ & $56 \mathrm{~Hz}$ \\
\hline $\begin{array}{c}\text { LLRF -VCO test } \\
\text { pulse }\end{array}$ & $5 \mathrm{~ms}$ & $3 \mathrm{~ms}$ & $45 \mathrm{~Hz}$ \\
\hline $\begin{array}{c}\text { Waveguide } \\
\text { Vacuum Fault }\end{array}$ & $1.9 \mathrm{~ms}$ & $3 \mathrm{~ms}$ & $75 \mathrm{~Hz}$ \\
\hline $\begin{array}{c}\text { Electronic Quench } \\
\text { Fault }\end{array}$ & $80 \mu \mathrm{s}$ & $3 \mathrm{~ms}$ & $400 \mathrm{~Hz}$ \\
\hline
\end{tabular}


secondary pulse. The initial gradient was $8 \mathrm{MV} / \mathrm{m}$. The secondary pulse was set to $3.3 \mathrm{MV} / \mathrm{m}$ in order to avoid saturating the klystron or initiating a secondary arc.

Figures 3 and 4 are examples of the forward power required to maintain a stable gradient after an arc on the cavity side of a cold window. In Figure 3 the secondary pulse was applied $20 \mathrm{~ms}$ after the arc event. In Figure 4 the secondary pulse was applied $500 \mathrm{~ms}$ after the arc event.

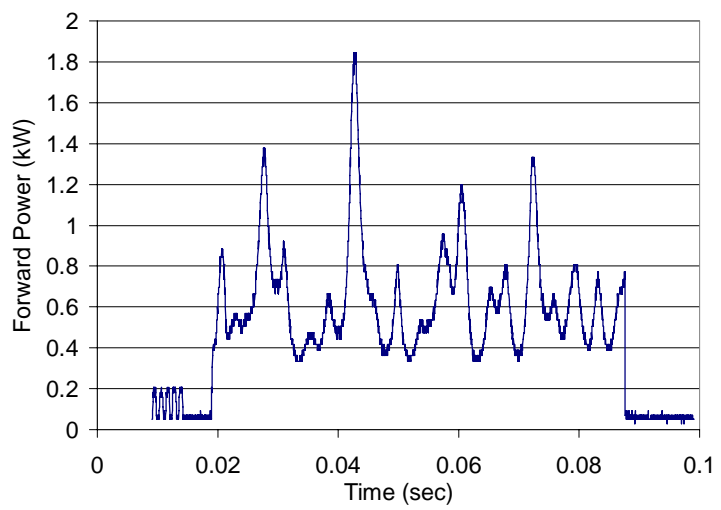

Figure 3: Forward Power during test pulse which was initiated $20 \mathrm{~ms}$ after an arc on the cavity side of the cold window.

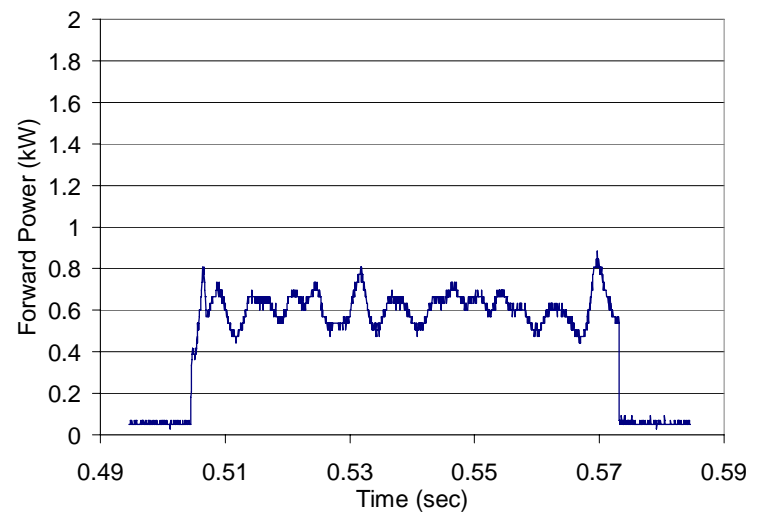

Figure 4: Forward Power during test pulse which was initiated $500 \mathrm{~ms}$ after an arc on the cavity side of the cold window.

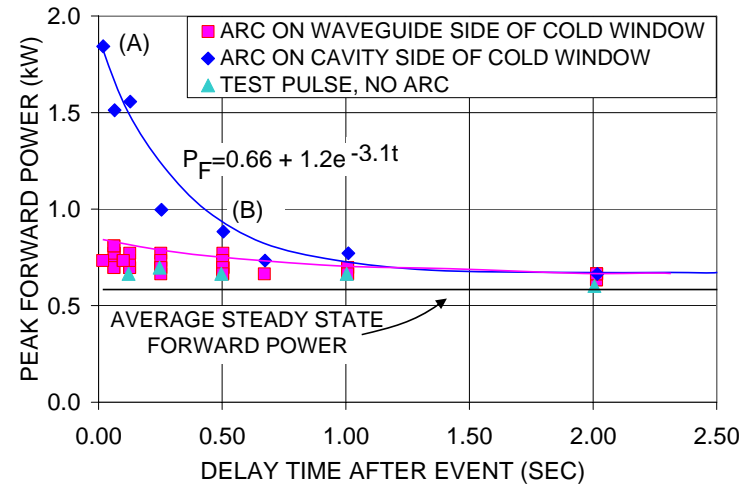

Figure 5: Summary of the peak to peak excursion of the forward power for different delays based on the type of arc event. Points (A) and (B) correspond to the data points shown in Figures 3 and 4 respectively.
Several events of each of the two types of arcs along with a series of events, which were initiated with a test pulse in the arc detector system, were recorded. A summary of the data is shown in Figure 5. The data plotted is the peak forward power required to maintain the stable gradient of $3.3 \mathrm{MV} / \mathrm{m}$ after the delay shown on the horizontal axis. Significant amounts of klystron dynamic reserve would be required if one were to try to reestablish gradient before the vibration modes have decayed.

Formula (1) provides a means to compare cavity frequency transients that were recorded with the cavity resonance monitor, to forward power transients, that were observed during the closed loop gradient control experiment [4]. This analysis is performed in detail in reference [3]. The calculated power was consistent with experimental results shown in Figure 5. In this formula $f$ is the frequency of the applied RF power, $\delta f$ is the difference between $f$ and the cavity frequency, $Q_{L}$ is the loaded-Q of the cavity, $E$ is the gradient in $\mathrm{V} / \mathrm{m},(\mathrm{r} / \mathrm{Q})$ is the geometrical shunt impedance in $\Omega / \mathrm{m}$ and $\mathrm{L}$ is the cavities electrical length.

$$
P_{\text {Klystron }}=\frac{E^{2} Q_{L}}{4} \frac{L}{(r / Q)}\left[\left(\frac{1}{Q_{L}}\right)^{2}+\left(2 \frac{\delta f}{f}\right)^{2}\right]
$$

\section{CONCLUSION}

There are cavity vibration modes that, coupled with beam-loaded klystron power margins, limit the recovery of CEBAF cavities after an arc event. The most likely source of the vibration excitation is the dynamic Lorentz force detuning which occurs when the cavity gradient is rapidly reduced by an arc event. This effect is substantially worse for an arc which occurs on the cavity side of the cold window where the gradient decays in less than $100 \mu \mathrm{s}$. Using the existing RF system, one would probably have to wait for at least $500 \mathrm{~ms}$ prior to applying $\mathrm{RF}$ and about 1.5 seconds prior to loading the system with beam.

\section{REFERENCES}

[1] P. Kneisel, T. Powers, "Response of CEBAF's cold RF-window to operation in FE-regime of a cavity," CEBAF-TN-94-029.

[2] T. Powers, L. Phillips, C. Reece, P. Kneisel, V. Nguyen, "RF window arcing studies update: a comparison of results from cryomodule 17 and vertical cavity testing," CEBAF-TN-94-059.

[3] T. Powers, D. Curry, K. Davis, L. King, M. Tiefenbach, "Waveguide Arc Restrike Tests Results," JLAB-TN-04-039.

[4] L. Merminga, J. Delayen, "On the Optimization of Qext Under Heavy Beam Loading and In the Presence of Microphonics, CEBAF-TN96-022. 